\title{
La fascination pour Alexandre le Grand dans les littératures européennes ( $\mathrm{X}^{e}-\mathrm{XVI} \mathrm{e}^{e}$ siècle). Réinventions d'un mythe, sous la direction de Catherine GAULLIER- BOUGASSAS
}

\section{Graziella Pastore}

\section{(2) OpenEdition \\ Journals}

\section{Edizione digitale}

URL: https://journals.openedition.org/studifrancesi/12368

DOI: 10.4000/studifrancesi. 12368

ISSN: 2421-5856

\section{Editore}

Rosenberg \& Sellier

\section{Edizione cartacea}

Data di pubblicazione: 1 avril 2018

Paginazione: 103-104

ISSN: 0039-2944

\section{Notizia bibliografica digitale}

Graziella Pastore, "La fascination pour Alexandre le Grand dans les littératures européennes ( $x^{e}$-xvI ${ }^{e}$ siècle). Réinventions d'un mythe, sous la direction de Catherine gauluIER-BOugassas», Studi Francesi [Online], 184 (LXII | I) | 2018, online dal 04 juillet 2018, consultato il 15 novembre 2021. URL: http:// journals.openedition.org/studifrancesi/12368; DOI: https://doi.org/10.4000/studifrancesi. 12368

Questo documento è stato generato automaticamente il 15 novembre 2021.

\section{(†)

Studi Francesi è distribuita con Licenza Creative Commons Attribuzione - Non commerciale - Non opere derivate 4.0 Internazionale. 


\title{
La fascination pour Alexandre le Grand dans les littératures européennes ( $\mathrm{C}^{e}$ - $\mathrm{XVI} \mathrm{e}^{e}$ siècle). Réinventions d'un mythe, sous la direction de Catherine
} GAULLIER-BOUGASSAS

\author{
Graziella Pastore
}

\section{NOTIZIA}

La fascination pour Alexandre le Grand dans les littératures européennes ( $\mathrm{X}^{e}-\mathrm{XVI} \mathrm{I}^{e}$ siècle). Réinventions d'un mythe, sous la direction de Catherine GAULLIER-BOUGASSAS, Turnhout, Brepols, 2014, « Alexander redivivus» 5, 4 voll., 2572 pp.

1 Quinta uscita della prolifica collezione Alexander redivivus, i quattro volumi che compongono quest'opera costituiscono un'analisi organica e ben documentata di come in tutta Europa la trasmissione e la rielaborazione dei testi antichi siano alla base di un florilegio di opere letterarie che reinventano la figura di Alessandro Magno.

2 Lo studio prende in considerazione opere appartenenti alle letterature dell'Europa occidentale (medio-latina, francese, italiana, iberica e aljamiada, ebraica, inglese, tedesca, olandese e scandinava) e dell'Europa orientale (ceca, serba, russa, bizantina e armena), composte fra il x e la prima metà del XVı secolo. Questo esteso corpus testuale comprende opere edite o ancora inedite, note o inesplorate, sia dedicate esclusivamente alla vita leggendaria del condottiero macedone o dei suoi antenati, sia testi che integrano episodi della vita di Alessandro all'interno di una trattazione storica o didattica più ampia, sia opere che accordano una particolare attenzione ad alcuni degli episodi più salienti della vita di Alessandro. 
3 L'introduzione (vol. 1, pp. 7-23) precisa la struttura generale dei quattro volumi e l'intento dell'opera, prefigurando un'impostazione diversa rispetto alla messe di opere precedentemente dedicate alla figura di Alessandro, in particolare alle opere fondatrici di George Cary (The Medieval Alexander, Cambridge, 1956) e di David J.A. Ross (Alexander Historiatus, a Guide to Medieval Illustrated Alexander Literature, London, 1963) che non abbracciano l'integralità del panorama letterario europeo né la sua evoluzione. È messa in risalto in particolare la volontà di affrontare lo studio della reinvenzione della figura di Alessandro nelle diverse aree linguistiche e letterarie alla luce della tradizione di studi sulla costituzione di uno spazio letterario comune europeo, inaugurata dalla opera pionieristica di Ernst Robert Curtius (vol. 1, p.10). Queste prime pagine sono l'occasione per ripercorrere brevemente l'origine dei primi scritti storici su Alessandro e del Roman d'Alexandre dello Pseudo-Callistene, fino al x sec., momento identificato come l'inizio della reinvenzione del mito di Alessandro nelle letterature europee.

4 Lo studio è articolato in cinque grandi sezioni ripartite nei primi tre volumi, ognuna delle quali è preceduta da una introduzione ed internamente suddivisa secondo la lingua di composizione dei testi.

5 I sezione (vol. 1, pp. 25-105) : «Panorama des littératures européennes sur Alexandre ( $\mathrm{x}^{\mathrm{e}}-\mathrm{XVI}^{\mathrm{e}}$ siècle)». Breve rassegna che raccoglie le informazioni principali e le grandi tendenze dell'insieme dei testi che trattano della figura di Alessandro Magno fra il $\mathrm{x}$ e il XVI secolo; le schede che la compongono introducono le opere che saranno discusse in maniera più approfondita nelle sezioni successive e in particolare nel volume 4.

6 II sezione (vol. 1, pp.107-678): «La traduction et l'adaptation aux sources de la création sur Alexandre dans les littératures européennes». Un insieme di quindici studi che ripercorrono le modalità di traduzione e adattamento dei testi antichi all'origine del corpus europeo di Alessandro con un'attenzione prevalentemente stilistica e linguistica (in particolare : L'Alexandreis" de Gautier de Châtillon di Jean-Yves TILLIETTE; L'Alexandre en français et ses univers littéraires multiples di Catherine GAULLIER-BOUGASSAS, Hélène BELLON-MÉGUELLE et Janet VAN DER MEULEN; Lettres anglaises dans une culture plurilingue di Margaret BRIDGES; Langues et genres littéraires de l'Alexandre italien di Michele CAMPOPIANO; Aspects linguistiques et littéraires de l'Alexandre russe di Elena KOROLEVA).

7 III sezione (vol. 2, pp. 679-1268): «Le pouvoir royal d'Alexandre: littérature et politique. Les auteurs, leurs mécènes et leurs publics». Dodici studi dedicati a mettere in luce l'assimilazione più o meno intensa di Alessandro ai valori politici, etici e religiosi nelle diverse letterature europee (in particolare: Les exemplarités politiques de l'Alexandre français et leurs mises à l'épreuve di Catherine GAULLIER-BOUGASSAS e Hélène BELLON-MÉGUeLLE; Alexandre le Grand et les idéaux politiques de la cour de Castille et Léon di Amaia ARIZALETA, Hugo Ó. BIZZARRI e Fernanda NUSSBAUM; L'image royale changeante d'Alexandre dans le contexte du Saint Empire di Marie-Sophie MASSE e Christophe THIERRY).

IV sezione (vol. 3, pp. 1269-1707) : « Alexandre scientifique et aventurier. Un imaginaire de la connaissance savante et des lointains exotiques». Dieci studi che mettono in evidenza i diversi tentativi di proiettare sulla figura di Alessandro Magno il desiderio di sapere, di sperimentazione scientifica, e l'apertura verso gli spazi esotici (in particolare : Alexandre explorateur des merveilles de l'Orient dans l'“Historia de preliis" di Alexandru CızEK ; Rêves de connaissance et d'exotisme : l'Alexandre aventurier en français di 
Catherine GAULLIER-BOUgASSAS e Hélène BELLON-MÉGUeLle; Voyages de l'Alexandre grec: le goût des merveilles et sa mise en question di Corinne JOUANNo).

9 V sezione (vol. 3, pp. 1709-1813): "Alexandre et la formation d'un espace littéraire européen». Quest'ultima sezione si focalizza sui ritmi rispettivi delle diverse letterature europee, sulla fortuna di ciascuna di esse al di fuori del proprio ambito linguistico, sugli ambienti di produzione e di ricezione delle opere, sui rapporti tra eventi storici e finzione letteraria e infine sulle ragioni del successo della figura di Alessandro Magno nello spazio letterario europeo, con le sue contraddizioni ed ambiguità.

Una breve conclusione (vol. 3, pp. 1815-1823) precede un'estesa bibliografia (vol. 3, pp. 1827-1978) che contiene i riferimenti ai manoscritti, alle edizioni antiche e moderne ed agli studi critici utilizzati nel corso dell'analisi. Il terzo volume è infine completato dall'indice delle opere e degli autori (pp.1979-2020) e dall'indice generale dei tre volumi (pp. 2021-2034).

11 Il volume 4 costituisce uno strumento di lavoro a parte, contenente il repertorio dei principali testi del corpus europeo, ripartiti secondo la loro lingua di redazione. Le schede del corpus sono presentate secondo lo schema: 1 . edizione o manoscritto di riferimento; 2. autore; 3 . data di redazione; 4. area di redazione ed eventuale committente/dedicatario ; 5 . forma letteraria e lista degli episodi narrati ; 6 . fonti ; 7 . elenco dei testimoni manoscritti o a stampa; 8. eventuali traduzioni, adattazioni o continuazioni ; 9. bibliografia specifica.

12 Frutto della collaborazione interdisciplinare di diciassette ricercatori coordinati da Catherine GAULLIER-BOUGASSAS, questi quattro volumi costituiscono una summa preziosa ed aggiornata della fortuna della figura di Alessandro Magno attraverso sei secoli di letterature europee. 